\title{
Каталог Planck - параметры переменности АЯГ на миллиметровых волнах
}

\author{
А.Е. Вольвач ${ }^{1}$, М.Г. Ларионов ${ }^{2}$, Л.Н. Вольвач ${ }^{1}$ \\ 1 ФГБУН “Крымская астрофизическая обсерватория РАН”, Научный, Крым, 298409 \\ volvach@craocrimea.ru \\ 2 Астрокосмический центр Физического института им. П.Н. Лебедева РАН, Москва, Россия
}

Поступила в редакцию 15 ноября 2017 г.

\begin{abstract}
Аннотация. С использованием радиотелескопа РТ-22 KрAО на частоте 36.8 ГГц выполнены наблюдения полной выборки 104 ярких активных ядер галактик из каталога Planck. Bce 104 источника анализируемого списка имеют потоки на 36.8 ГГц более 1 Ян и являются известными объектами, зафиксированными в каталогах радиоисточников. Полученные наблюдательные данные подтверждают выводы о том, что уровень переменности плотности потока излучения АЯГ максимальный на миллиметровых волнах по сравнению с другими частотами радиодиапазона. Изменение индекса переменности АЯГ, возможно, происходит из-за систематического падения спектральной плотности их излучения при переходе в инфракрасный диапазон длин волн. Изменение индекса переменности АЯГ в миллиметровом диапазоне не противоречит предположению о том, что переменность потока вызвана внутренними причинами в двойных системах из сверхмассивных черных дыр, находящихся на стадии эволюции, близкой к слиянию.

THE PLANCK CATALOGUE - PARAMETERS OF AGN VARIABILITY AT MILLIMETER WAVELENGTHS, by A.E. Volvach, M.G. Larionov, L.N. Volvach. Observations of the full sample of 104 bright active galactic nuclei from the Planck catalogue have been carried out with the RT-22 radio telescope $(\mathrm{CrAO})$ at a frequency of $36.8 \mathrm{GHz}$. All 104 sources from the analyzed list have fluxes at $36.8 \mathrm{GHz}$ more than $1 \mathrm{Jy}$ and these are the known objects recorded in the catalogues of radio sources. The obtained observation data prove the conclusions that the level of flux density variability of AGN emission is maximal at millimeter wavelengths compared to other frequencies in the radio range. Variations in variability index of AGN presumably occur due to the systematic increase in spectral density of their emission as a result of transition to the infrared wavelength range. Variations in variability index of AGN in millimeter wavelengths are not in contrast to the assumption that the flux variability is caused by inner reasons in binary systems from supermassive black holes that are at the evolution stage close to merging.
\end{abstract}

Ключевые слова: активные ядра галактик, радиоизлучение, переменность плотности потока

\section{1 Введение}

В результате работы двух космических миссий WMAP и Planck было обнаружено большое количество объектов галактического и внегалактического происхождения, которые различались по спек- 
тральным характеристикам и признакам переменности (Планк, 2011). Предметом исследований являются активные ядра галактик (АЯГ), обнаружившие предельные значения индексов переменности, как источники, перспективные для наблюдений по программе "Радиоастрон” методом многочастотного синтеза, наземно-космические наблюдения которых планируется начать во второй половине 2018 года. Дополнительная цель исследований - установить, все ли объекты списка являются известными и зафиксированными в каталогах АЯГ, мониторинг которых ведется в Симеизе уже долгие годы, и провести соответствующий анализ их переменности, так как диаграмма направленности космического радиотелескопа Planck не позволяла со $100 \%$ уверенностью идентифицировать все источники.

\section{2 Наблюдения}

В анализируемом списке присутствуют 104 источника, представляющие собой полную выборку АЯГ до уровня потока 1 Ян на частоте 36.8 ГГц, и расположенные севернее $-10^{\circ}$ по склонению. Список скомпилирован из данных каталога компактных источников ERCSC (Early Release Compact Sources Catalogue), полученных на 9 частотах в диапазоне от 20 до 857 ГГц в период с 13 августа 2009 г. по 6 июня 2010 г. (Планк, 2011). В списке содержится 33 квазара с высокой поляризацией (HPOs) co степенью оптической поляризации более $3 \%, 21$ объект с низкой степенью поляризации (LPOs), где поляризация менее 3\%, 21 блазар (BLOs), 19 обычных квазаров (QSOs), в которых поляризацию не удалось измерить, 9 радиогалактик (GALs) и один источник без классификации (J184915+67064). Большинство объектов имеют среднее значение спектральной плотности потока, измеренной в двух сетах, разделенных временем около 6 месяцев.

Наблюдения на 22-м радиотелескопе РТ-22 выполнялись на частоте 36.8 ГГц. Прием информации производился в режиме диаграммной модуляции с использованием приемной системы с полосой 1 ГГц. Среднее время усреднения одного значения антенной температуры от источника было (1200$1400)$ сек. При хороших погодных условиях предел выделения по потоку составлял 0.2 Ян. Рассматривались источники, имевшие уровень отношения сигнал/шум более 4. Шкала по потоку была привязана к источнику с постоянной плотностью потока - DR 21. В качестве вторичных калибраторов использовались источники NGC 7027, 3С274, 3С84.

\section{3 Анализ данных}

Для исследования переменности 104 АЯГ обзора Planck была использована методика, подобная изложенной в работе (Мингалиев и др., 2009). Использовался индекс переменности и ошибка индекса переменности, вычисляемые по формулам:

$$
\begin{gathered}
V_{v e r}=\left(\frac{S_{\max }-S_{\min }}{S_{\min }}\right) \cdot 100, \\
\Delta V_{v e r}=\frac{\left|S_{\max } \Delta S_{\min }-S_{\min } \Delta S_{\max }\right|}{S_{\min }^{2}},
\end{gathered}
$$

где $S_{\max }$ и $S_{\min }$ - максимальное и минимальное значения потоков источника за период наблюдений, а $\Delta S_{\max }$ и $\Delta S_{\min }$ - ошибки значений потоков.

Интерполированные на частоту 36.8 ГГц значения потоков источников, выделенных в обзоре WMAP, являются усредненными за 5 лет наблюдений значениями, отнесенными на эпоху середины 2004 года. Данные, полученные в обсерватории Метсахови, датируются второй половиной 2009 года. Радиоисточники списка измерялись в Симеизе в первой половине 2015 года. Таким образом, максимальный интервал между наблюдениями составил около 11 лет. Этот период достаточный, чтобы проявилась долговременная переменность потока излучения в большинстве АЯГ.

Из анализа долговременной переменности потока излучения наиболее ярких представителей АЯГ, выполненного на основе данных обсерваторий Симеиза, Метсахови и Мичиганского университета (США), полученных в диапазоне частот (4-37) ГГц, следует, что как раз в указанном временном 
Таблица 1. Источники обзора Planck с большим индексом переменности

\begin{tabular}{c|c|c|l|c}
\hline № & Имя & Имя & Отождествление & $\begin{array}{c}\text { Поток на 36.8 ГГц, } \\
\text { псредненный по времени }\end{array}$ \\
\hline 1 & 1950.0 & 2000.0 & & 1.75 \\
2 & $0224+671$ & $0228+6721$ & GB6 0224+671 & 1.65 \\
3 & $0306+102$ & $0309+1029$ & PKS 0306+1029 & 4.2 \\
4 & $0420-014$ & $0359+5057$ & NRAO150 & 6.0 \\
5 & $0507+179$ & $0510+0120$ & OA129 & 1.8 \\
6 & $0552+398$ & $0555+3948$ & GB6 0507+179 & 2.0 \\
7 & $0735+178$ & $0738+1742$ & DA193 & 1.3 \\
8 & $0923+392$ & $0927+3902$ & 4C 39.25 & 5.2 \\
9 & $1055+018$ & $1058+0133$ & OL093 & 5.0 \\
10 & $1324+224$ & $1327+2210$ & GB6 1324+224 & 1.3 \\
11 & $1418+546$ & $1419+5423$ & OQ530 & 1.3 \\
12 & $1642+690$ & $1642+6856$ & GB6 1642+690 & 2.2 \\
13 & $1741-038$ & $1743-0350$ & PKS 1741-038 & 2.9 \\
14 & $1954+513$ & $1955+5131$ & GB6 1954+513 & 1.2 \\
15 & $2007+776$ & $2005+7752$ & S52007+77 & 1.1 \\
16 & $2136+141$ & $2139+1423$ & GB6 2136+141 & 1.4 \\
17 & $2227-088$ & $2229-0832$ & AT20G2229-083 & 2.8 \\
\hline
\end{tabular}

\section{Подписи к таблице:}

1 колонка - номер источника,

2 колонка - формат имени источника в нотации 1950.0,

3 колонка - формат имени источника в нотации 2000.0,

4 колонка - отождествление объекта с данными других радиокаталогов,

5 колонка - плотность потока источника на частоте 36.8 ГГц, усредненного по времени

диапазоне могут заключаться прецессионные периоды двойных сверхмассивных черных дыр (СЧД), находящихся в центральных областях АЯГ (Вольвач и др., 2016).

В таблице 1 приведен список источников обзора Planck, имеющих максимальные значения индекса переменности. Эти объекты являются перспективными для включения в программу наблюдений методом многочастотного синтеза проекта "Радиоастрон”.

Из наблюдавшихся нами 104 объектов 24 источника не были обнаружены в обзоре WMAP. Это может свидетельствовать в пользу того, что значительная доля этих АЯГ имеет большие индексы переменности потока излучения и часть их не видно в обзоре WMAP. Часть из них находится существенно выше уровня выделения источников в обзоре WMAP. К ним в первую очередь относится источник PKS 0059+581, поток которого существенно превышает порог выделения в обзоре WMAP.

Значительные уменьшения плотности потока излучения зафиксированы нами в объектах 0355+508, 0415+379, S0528+134. Последний является одним из удаленных АЯГ (Ларионов и др., 1971). Подобные изменения потоков в этих источниках фиксировались и ранее на основе результатов длительных мониторингов в широком диапазоне длин волн (Вольвач и др., 2014). Один из самых мощных интерферометрических калибраторов, S0528+134, отсутствует в обзоре WMAP, хотя в наземных наблюдениях его поток на частотах 22 и 36 ГГц временами превышает 6 Ян. В этом АЯГ S0528+134 за все время наблюдений с момента открытия при проведении симеизского обзора (Ларионов и др., 1971) были отмечены рекордные изменения потока на величину в более 10 раз. Картина сильной переменности плотности потока видна в объектах 0923+392, 1741-038, которые также отсутствуют в списке WMAP. 
Отдельно стоит остановиться на блазаре 3C454.3, который является ярчайшим представителем класса объектов АЯГ. Диапазон изменения его плотности потока превышает шестикратную величину (Вольвач и др., 2007). В объекте регистрируются многочисленные вспышки со значительными изменениями потока излучения. В последние два года это АЯГ находится практически в состоянии непрерывной вспышки, что может указывать на серьезные процессы, происходящие в этой двойной системе из СЧД и связанные с прохождением массивного компаньона через аккреционный диск (АД) центральной СЧД.

Кроме того, в анализируемом списке АЯГ-источников обзора Planck присутствует еще ряд объектов, перспективных для наблюдений наземно-космической миссией "Радиоастрон” и будущей миссией “Миллиметрон”. Такие радиоисточники имеют максимумы в спектральной плотности излучения, расположенные на миллиметровых волнах. Они содержат в своей структуре более компактные образования в сравнении с теми АЯГ, которые имеют максимумы в спектрах на сантиметровых волнах.

\section{4 Статистика по количеству АЯГ}

В работе (Вольвач и др., 2017) высказывалось предположение, что наиболее яркие АЯГ являются тесными двойными системами из СЧД. Это свойство, а также то, что система находится на стадии эволюции, близкой к слиянию, приводит к тому, что компаньон центральной СЧД перемещается по орбите, расположенной в среде с повышенной плотностью аккрецирующего вещества, пересекает в перицентре АД центральной СЧД. В момент пересечения АД компаньоном и наблюдаются вспышечные явления. В работе (Вольвач и др., 2007) было показано, что размеры орбиты тесных двойных систем (ТДС) соизмеримы с диаметром АД.

В соответствии с изложенной моделью АЯГ мы должны наблюдать их количество, соответствующее нашим представлениям об эволюции массивных галактик во Вселенной, по которым в центрах массивных галактик должны быть СЧД. Таких галактик во Вселенной около $10^{10}$.

Согласно наблюдениям, плотность радиоисточников на небе на частоте 20 ГГц с потоками более 1 Ян равна 25 в одном стерадиане (Садлер и др., 2006). Из них доля тех, которые имеют неубывающие в сторону коротких волн плотности потоков излучения, примерно $50 \%$. Отсюда следует, что на всей небесной сфере имеется около 150 АЯГ с потоками больше 1 Ян на этой частоте. Плотность числа источников с так называемыми “плоскими” спектрами убывает с потоком. Так, если для потоков более 1 Ян их пропорция составляет около $50 \%$ от общего числа объектов, то к потоку 0.1 Ян это соотношение в два раза ниже, то есть $25 \%$. Это означает, что доля АЯГ в общем числе радиоисточников ощутимо падает с уменьшением потока.

С другой стороны, мы не располагаем достоверными данными о том, на каких красных смещениях АЯГ уже сформировались. Известны сильные АЯГ с красными смещениями $z \geq 2$ (например, $\mathrm{S} 0528+134, \mathrm{Nimfa).} \mathrm{Это} \mathrm{означает,} \mathrm{что} \mathrm{на} \mathrm{этих} z$ в центральных областях активных галактик уже существуют черные дыры с массами $M \geq 10^{9} M_{\mathrm{o}}$. В активной фазе такие объекты имеют потоки более 10 Ян, что может указывать на то, что мы способны регистрировать все ТДС из СЧД до красных смещений $z=(5-6)$, но нам не известны АЯГ в массовом количестве, имеющие такие красные смещения. Тогда, скорее всего, АЯГ формировались на $z=(2-3)$ и мы наблюдаем все АЯГ, проявляющие повышенную активность.

Для оценки полного числа АЯГ на небесной сфере еще необходимо знать среднее значение угла раствора конуса излучения. В подавляющем большинстве наиболее ярких АЯГ угол между выбросами (“джетом”) и направлением на наблюдателя не превышает нескольких градусов. Если принять для таких углов ограничение в (5-6) градусов, то мы фактически наблюдаем только $10^{-2}$ часть от общего числа таких источников на небе. Следовательно, их общее число должно быть $1.5 \cdot 10^{4}$, что составляет только около $10^{-6}$ от общего числа массивных галактик во Вселенной $\left(10^{10}\right)$. Именно короткое время жизни АЯГ (не более $10^{4}$ лет) приводит к тому, что такие источники живут $10^{-6}$ часть времени от возраста Вселенной и составляют такую же долю от общего числа массивных галактик во Вселенной. Поэтому мы и наблюдаем их как 150 ярких представителей АЯГ, а их общее число на небе в сто раз больше из-за узкого конуса излучения таких источников. 
Таким образом, статистические данные указывают на то, что из-за узкой направленности излучения мы наблюдаем около $1 \%$ АЯГ, представляющих собой ТДС из СЧД и находящихся на стадии эволюции, близкой к слиянию. Их доля по отношению к общему числу массивных галактик во Вселенной незначительна и составляет величину $10^{-6}$, что определяется их непродолжительным временем жизни по отношению к возрасту Вселенной.

\section{5 Заключение}

Получены новые данные, свидетельствующие о том, что АЯГ на миллиметровых волнах обнаруживают максимальный уровень переменности плотности потока излучения по сравнению с другими частотами радиодиапазона. Некоторые из АЯГ показывают признаки значительного изменения плотности потока излучения, поэтому при анализе их переменности на больших временных шкалах могут выпадать из соответствующей выборки. Увеличение индекса переменности АЯГ в миллиметровом диапазоне длин волн не противоречит предположению о том, что наиболее яркие представители АЯГ представляют собой тесные двойные системы из сверхмассивных черных дыр, находящиеся на стадии эволюции, близкой к слиянию.

Наблюдаемое незначительное число ярких представителей АЯГ по отношению к общему числу массивных эллиптических галактик может объясняться их малым временем существования как тесных систем из СЧД и механизмом излучения, происходящего из узкого конуса по направлению к наблюдателю. С уменьшением потока доля АЯГ по отношению к общей плотности радиоисточников падает и составляет четверть на уровне потока 0.1 Ян.

\section{Литература}

Вольвач А.Е., Вольвач Л.Н., Ларионов М.Г., Аллер Х.Д., Аллер М.Ф. // Астрон. журн. 2007. Т. 84. № 6. C. 503.

Вольвач А.Е., Кутькин А.М., Ларионов М.Г., Вольвач Л.Н. и др. // Астрон. журн. 2014. Т. 91. №. 2. C. 122 .

Вольвач и др. (Volvach A.E., Kardashev N.S., Larionov M.G., and Volvach L.N.) // Astron. Reports. 2016. V. 60. N. 7. P. 621.

Вольвач А.Е., Ларионов М.Г., Вольвач Л.Н., Ларионов Г.М. // Астрон. журн. 2017. Т. 94. № 11. C. 950.

Ларионов М.Г., Горшков А.Г., Попов М.В., Моисеев И.Г. // Астрон. циркуляр. 1971. Т. 665. С. 1.

Мингалиев М.Г., Сотникова Ю.В., Кардашев Н.С., Ларионов М.Г. // Астрон. журн. 2009. Т. 86. ․ 4. C. 1.

Планк (Planck Collaboration) // Astron. Astrophys. 2011. V. 536. id. A7.

Садлер и др. (Sadler E.M., Ricci R., Ekers R.D., et al.) // Mon. Not. Roy. Astron. Soc. 2006. V. 371. Issue 2. P. 898. 